\title{
De novo SOX10 nonsense mutation in a patient with Kallmann syndrome and hearing loss
}

\author{
Kirsi Vaaralahti ${ }^{1}$, Johanna Tommiska ${ }^{1,2}$, Vallo Tillmann ${ }^{3,4}$, Natalja Liivak ${ }^{5}$, Johanna Känsäkoski ${ }^{1}$, Eeva-Maria Laitinen ${ }^{1,2}$ and \\ Taneli Raivio ${ }^{1,2}$
}

K allmann syndrome (KS; Mendelian Inheritance in Man (MIM)\# 147950), a combination of congenital hypogonadotropic hypogonadism (MIM\# 146110) and decreased/ absent sense of smell, results from disturbed migration of gonadotropin-releasing hormone neurons along the olfactory sensory neurons from the olfactory placode to the hypothalamus. The reproductive phenotype may vary from severe hypogonadism to reversal of hypogonadotropism later in life. Patients may also have additional nonreproductive features, which include cleft lip/palate, dental agenesis, syndactyly, limb anomalies, renal agenesis, scoliosis, mirror movements, and deafness (1).

Very recently, Pingault et al. described a high prevalence of SOX10 mutations in patients with KS and deafness (2). SOX10 is a transcription factor involved in many cellular and developmental processes, especially in the development and differentiation of the neural crest (3). Mutations in SOX10 are known to cause Waardenburg syndrome (WS), which is a rare developmental disorder characterized by pigmentation abnormalities and deafness (specifically type 4C, which combines Waardenburg and Hirschsprung disease MIM\# 613266; its neurological variant PCWH MIM\#609136; and also WS type 2E MIM\# 611584) (4-6). Involvement of SOX10 mutations in KS was suspected when a high frequency of olfactory bulb agenesis was found among patients with WS. Indeed, seven novel loss-of-function mutations (four missense mutations, one frameshift mutation, one splice-site mutation, and one mutation located in the initiation codon) in SOX10 were found among altogether 103 patients with KS (2). Notably, six of these seven mutation carriers had a hearing impairment suggesting that SOX10 mutations should be screened among patients with KS and deafness (2). We further investigated the role of SOX 10 in KS by screening 20 patients with $\mathrm{KS}$ with special interest in four patients with hearing impairment.

The clinical features of 18 Finnish male subjects with KS have been reported in Laitinen et al. (7). In addition, one female patient with $\mathrm{KS}$ with a history of spontaneous puberty followed by secondary amenorrhea, congenital anosmia, absent olfactory bulbs in the magnetic resonance imaging, and low estradiol (E2) in the setting of normal gonadotropin levels, thus representing mild gonadotropin-releasing hormone deficiency, was included. Three of the male subjects had hearing impairment.
One had bilateral hearing impairment and other CHARGE syndrome (coloboma, heart defects, choanal atresia, retarded growth and development, genital abnormalities, and ear anomalies; MIM\# 214800) associated features (cleft lip and palate, unilateral microphthalmia and coloboma, left facial nerve palsy, cup-shaped ears, upper body muscular atrophy, and hypoplastic semicircular canals), the other had bilateral sensorineural hearing loss, and the third had unilateral hearing loss. In addition, one patient had CHARGE syndrome-associated features without hearing impairment (cup-shaped ears and upper body muscular atrophy). The fourth patient with KS and hearing loss was from Estonia. His phenotypic features are described below.

None of the subjects has been found to carry mutations in the seven genes known to underlie KS (KAL1, FGFR1, FGF8, PROK2, PROKR2, CHD7, WDR11), and no intragenic deletions were found in CHD7 or FGFR1 multiplex ligationdependent probe amplification assays (MRC-Holland, Amsterdam, the Netherlands) as described in Laitinen et al. (7). This study was performed with appropriate permissions from the Ethics Committee (E7) of the Helsinki University Central Hospital and from each university hospital in Finland, and from the Ethics Review Committee on Human Research of the University of Tartu. All subjects obtained and signed the written consent. Genomic DNA from peripheral blood leukocytes of the subjects was extracted, and the coding exons and exon-intron boundaries of SOX10 (NM_006941.3) were PCR amplified and screened by direct sequencing. Primer sequences and PCR conditions are available on request. Mutation was confirmed from a second PCR product.

One male patient with KS and hearing loss carried a heterozygous nonsense mutation c.184G $>\mathrm{T}$ (p.Glu62X) in the first coding exon of SOX10. This mutation was confirmed as de novo (absence of the mutation was verified by sequencing in both parents), and it was not present in 90 control individuals or reported in relevant databases (dbSNP:http://www.ncbi. nlm.nih.gov/projects/SNP/, 1000 Genomes Project:http:// www.1000genomes.org/, NHLBI Exome Variant Server: http:// evs.gs.washington.edu/EVS/, Leiden Open Variation Database: http://grenada.lumc.nl/LOVD2/WS/home.php?select_ $\left.\mathrm{db}^{1 / 4} \mathrm{SOX} 10\right)$. The Glu62X mutation most likely activates the

\footnotetext{
'Institute of Biomedicine/Physiology, University of Helsinki, Helsinki, Finland; ${ }^{2}$ Children's Hospital, Helsinki University Central Hospital (HUCH), Helsinki, Finland; ${ }^{3}$ Department of Pediatrics, University of Tartu, Tartu, Estonia; ${ }^{4}$ Children's Clinic, Tartu University Hospital, Tartu, Estonia; ${ }^{5}$ Tallinn Children's Hospital, Tallinn, Estonia. E-mail: Taneli Raivio (taneli.raivio@helsinki.fi)
}

Received 6 November 2013; accepted 24 January 2014; advance online publication 7 May 2014. doi:10.1038/pr.2014.60 
nonsense mediated decay RNA surveillance pathway and results in SOX10 haploinsufficiency (8).

At the age of $17 \mathrm{y}$, this proband was referred to endocrinologist for evaluation of delayed puberty. He did not have a history of cryptorchidism, and his family history was negative for delayed puberty, cleft lip or palate, Hirschsprung disease, and KS. Based on anosmia from history, lack of puberty (Tanner stage G1P2) and bilaterally small testes $(<3 \mathrm{ml})$ on examination, together with low circulating basal testosterone $(<0.69 \mathrm{nmol} / \mathrm{l})$, in association with inappropriately low gonadotropin levels (folliclestimulating hormone $0.54 \mathrm{mU} / \mathrm{ml}$, luteinizing hormone 0.15 $\mathrm{mU} / \mathrm{ml}$ ), the diagnosis of KS was set, and puberty was induced with monthly Sustanon injections. His karyotype was 46, XY.

At the age of $19 \mathrm{y}$, he participated in a study investigating KS in Estonia. He had bilaterally small testes $(<3 \mathrm{ml})$ and anosmia as assessed with standardized twelve item odor stick series (Burghart Messtechnik, Wedel, Germany). Magnetic resonance imaging examination revealed olfactory bulb aplasia. When specifically asked for, he admitted early graying of hair, reduced sweating, but no gastrointestinal problems. Clinical examination confirmed the presence of gray hairs. He did not have birth marks, vitiligo, café au lait spots, or any other pigmentation defects in the skin. He had broad nasal bridge, but no synophrys or hypoplasia alae nasi. His hearing loss, present from birth, was complete on the right side, and mild sensorineural hearing loss in high frequencies was noted in his left ear audiogram (Supplementary Figure S1 online). He has mild myopia, and, in ophthalmologic examination, his fundi were normal. He had normal number of teeth. His muscle strength seemed normal, but he reported problems with balance, and was not able to stand on one foot. His joints in upper and lower extremities were hypermotile.

Mutations in SOX10 are known to underlie WS4, PCWH, and WS2 neurocristopathies, which all share in common hearing loss and pigmentary anomalies (4-6). Our patient also reported early graying of hair, and one patient with KS in Pingault's series had premature whitening of hair (2). Accordingly, SOX10 is important for the development of melanocytes, structures of inner ear, enteric ganglia neurons, Schwann cells, and oligodendrocytes $(9,10)$. Studies with SOX10-null mutant mice show that underlying mechanism of this form of KS results from defects in embryonic development of olfactory ensheathing cells that are required for gonadotropin-releasing hormone neuron migration $(2,11)$.

SOX10 is the second neural crest-related gene implicated in the etiology of KS. Indeed, mutations in CHD7-a key gene in neural crest development (12) - have been shown to underlie $3-5 \%$ of KS cases $(7,13,14)$, and CHD7 mutations may account as much as one third of KS cases with deafness (1). Our current data, especially when combined with that of Pingault et al., confirm a significant proportion of loss-of-function mutations in SOX10 underlying deafness in patients with KS (2).

In conclusion, targeting the molecular genetic diagnostics of KS can be challenging and therefore even subtle phenotypic cues may be of great value in this process. Hearing impairment is not a very common feature in KS, and its presence should lead the pediatrician to search additional phenotypic cues related to mutations in genes implicated in the development of neural crest, $\mathrm{CHD} 7$ and SOX10.

Finally, it should be noted that there is a $50 \%$ chance to pass on the SOX10 gene defect to future children, who subsequently might present with a more severe SOX10-linked phenotype $(4,15)$. Although this risk has not yet been evaluated in a patient with KS with SOX10 mutations, such situation has been documented in patients with KS and CHD7 mutations $(7,14)$.

\section{SUPPLEMENTARY MATERIAL}

Supplementary material is linked to the online version of the paper at http://www.nature.com/pr

\section{STATEMENT OF FINANCIAL SUPPORT}

This work was supported by the Academy of Finland, the Finnish Foundation for Pediatric Research, the Helsinki University Central Hospital Research Funds (EVO), Emil Aaltonen Foundation, and the Sigrid Juselius Foundation.

Disclosure: The authors have nothing to disclose.

\section{REFERENCES}

1. Costa-Barbosa FA, Balasubramanian R, Keefe KW, et al. Prioritizing genetic testing in patients with Kallmann syndrome using clinical phenotypes. J Clin Endocrinol Metab 2013;98:E943-53.

2. Pingault V, Bodereau V, Baral V, et al. Loss-of-function mutations in SOX10 cause Kallmann syndrome with deafness. Am J Hum Genet 2013;92:707-24.

3. Wegner M. Secrets to a healthy Sox life: lessons for melanocytes. Pigment Cell Res 2005;18:74-85.

4. Pingault V, Bondurand N, Kuhlbrodt K, et al. SOX10 mutations in patients with Waardenburg-Hirschsprung disease. Nat Genet 1998;18:171-3.

5. Inoue K, Shilo K, Boerkoel CF, et al. Congenital hypomyelinating neuropathy, central dysmyelination, and Waardenburg-Hirschsprung disease: phenotypes linked by SOX10 mutation. Ann Neurol 2002;52:836-42.

6. Bondurand N, Dastot-Le Moal F, Stanchina L, et al. Deletions at the SOX10 gene locus cause Waardenburg syndrome types 2 and 4 . Am J Hum Genet 2007;81:1169-85.

7. Laitinen EM, Vaaralahti K, Tommiska J, et al. Incidence, phenotypic features and molecular genetics of KS in Finland. Orphanet J Rare Dis 2011;17:1750-72.

8. Inoue $\mathrm{K}$, Khajavi $\mathrm{M}$, Ohyama $\mathrm{T}$, et al. Molecular mechanism for distinct neurological phenotypes conveyed by allelic truncating mutations. Nat Genet 2004;36:361-9.

9. Breuskin I, Bodson M, Thelen N, et al. Sox10 promotes the survival of cochlear progenitors during the establishment of the organ of Corti. Dev Biol 2009;335:327-39.

10. Britsch S, Goerich DE, Riethmacher D, et al. The transcription factor Sox10 is a key regulator of peripheral glial development. Genes Dev 2001;15:66-78.

11. Barraud P, St John JA, Stolt CC, Wegner M, Baker CV. Olfactory ensheathing glia are required for embryonic olfactory axon targeting and the migration of gonadotropin-releasing hormone neurons. Biol Open 2013;2:750-9.

12. Siebert JR, Graham JM Jr, MacDonald C. Pathologic features of the CHARGE association: support for involvement of the neural crest. Teratology 1985;31:331-6.

13. Kim HG, Kurth I, Lan F, et al. Mutations in CHD7, encoding a chromatinremodeling protein, cause idiopathic hypogonadotropic hypogonadism and Kallmann syndrome. Am J Hum Genet 2008;83:511-9.

14. Jongmans MC, van Ravenswaaij-Arts CM, Pitteloud $\mathrm{N}$, et al CHD7 mutations in patients initially diagnosed with Kallmann syndrome-the clinical overlap with CHARGE syndrome. Clin Genet 2009;75:65-71.

15. Pingault V, Girard M, Bondurand N, et al. SOX10 mutations in chronic intestinal pseudo-obstruction suggest a complex physiopathological mechanism. Hum Genet 2002;111:198-206. 